\title{
IUFOST2006/743 CLSM - a powerful tool for structure design
}

\author{
A.M. Hermansson \\ SIK, the Swedish Institute for Food and Biotechnology, Box 5401, 40229 Göteborg, Sweden \\ amh@sik.se
}

Confocal laser scanning microscopy is becoming more and more important in food research of colloidal structures. The laserbeam can penetrate into a bulk sample, and excite the fluorescent dyes coupled to the object of interest such as fat, polysaccharides or proteins. This makes it possible to study dynamic events such as changes taking place during cooling, heating or flow and to obtain information of importance for process optimisation. The term confocal means that information is obtained from one plane and light from all other planes is excluded by shielding with a small pinhole. Adjacent optical sections can be obtained by moving the confocal plane, i.e. optical sectioning instead of physical sectioning. Information from the stack of planes can be used to construct three-dimensional images. Since the image information is directly assembled in a computer, CLSM images are very suitable for further image analysis.

The possibilities to study transient structures under dynamic conditions directly under the microscope give us a powerful scientific advantage for new approaches of structure design. The result provides us with information about structural transitions to be used in modelling and design of food materials with inherent functional properties. It will be demonstrated how the kinetics during gel formation can be followed directly in the CLSM and its significance for the final morphology and related properties. Examples will be shown, where more than one mechanism is involved such as gel formation in combination with phase separation or aggregation. One of the biggest challenges for the food industry is to make products that breakdown or fail in a pre-designed way. The way a structure deforms and breaks under stress is crucial for properties such as flow and fracture behaviour, sensory perception of structure, water release and mobility and release of active compounds that need to be bio-available in order to function. A new field of research has been initiated at by studies of failure of food and gel based products on the macro and micron scale by access to a tensile stage that can be mounted directly under the CLSM. In addition new developments of fluorescense recovery after photobleaching will provide new information about diffusion properties of water and fat in complex food products. 